\title{
Identification and analysis of pathogenic nsSNPs in human LSP1 gene
}

\author{
Hani Mohammed Ali ${ }^{1}$ \\ 1Department of Biological Science, Faculty of Science, King Abdulaziz University, Jeddah, Kingdom of Saudi Arabia; Email: \\ hmohammedali@kau.edu.sa; haniolfat@hotmail.com; *Corresponding author
}

Received September 11, 2019; Revised September 20, 2019; Accepted September 29, 2019; Published September 30 , 2019

\begin{abstract}
:
LSP1 (Lymphocyte-specific protein 1) protein plays an important role in neutrophil motility, fibrinogen matrix proteins adhesion, and trans-endothelial migration. Variation in the LSP1 gene is associated with leukemia and lymphomas in tumor cells of Hodgkin's disease and breast cancer. Despite extensive study on the human LSP1, a comprehensive analysis on the Single Nucleotide Polymorphism (SNPs) of the gene is not available. Therefore, it is of interest to identify, collect, store and analyze the SNPs of the LSP1 gene in relation to several known diseases. Hence, the SNP data (398 rsids) from dbSNP database was downloaded and mapped to the genomic coordinate of "NM_002339.2" transcript expressed by LSP1 (P33241). There were 300 nsSNPs with missense mutation in the dataset. Tools such as SIFT, PROVEAN, Condel, and PolyPhen-2 were further used to identify 29 highly deleterious or damaging on synonymous SNP (nsSNPs) for LSP1. These high confident damaging nsSNPs were further analyzed for disease association using SNPs \& GO tool. SNPs of the gene such as nsSNPs C283R, G234R, Y328D and H325P showed disease association with high prevalence.
\end{abstract}

Keywords: SNP; Lymphocyte-specific protein; computational analysis; F-actin binding protein; neutrophil actin dysfunction

\section{Background:}

Human LSP1 (lymphocyte specific protein 1) gene encodes an intracellular F-actin binding protein, recently renamed as leukocyte specific protein. The protein is expressed in lymphocytes, macrophages, neutrophils, and endothelium and regulates adhesion to fibrinogen matrix proteins, neutrophil motility, and transendothelial migration. Due to alternative splicing there are multiple transcript variants which encodes different isoforms. Highest expression of this gene in spleen (RPKM 60.6), appendix (RPKM 43.3) and other tissues [1, 2] is known. LSP1 is found in plasma membrane internal surface of the, the cytoplasm, and is thought to mediate cytoskeleton-driven responses in activated leukocytes that involve receptor capping, cell-cell interactions and cell motility [3]. Lymphocyte specific protein 1 modulates leukocyte populations in resting and inflamed peritoneum [2]. The LSP1 protein is detected in leukemia and lymphomas in tumor cells of
Hodgkin's disease and breast cancer [4]. The motility of melanoma cell is inhibited even at low level of LSP1 expression [5]. Many research showed identifying the deleterious effectiveness and disease associated mutations, thus predicting the pathogenic nsSNPs in correlation to their functional and structural damaging properties [6-9]. Computational studies provide an efficient platform for analysis of genetic mutations for their pathological consequences and in determining their underlying molecular mechanism [10-11]. Single nucleotide polymorphism (SNPs) is a common genetic variations contributing greatly towards the phenotypic variations in the populations. SNPs can alter the functional consequences of proteins. In the coding region of gene, SNPs may be synonymous, non-synonymous (nsSNPs) or nonsense. Synonymous SNPs changes the nucleotide base residue but does not change the amino acid residue in protein sequence due to degeneracy of genetic code. The nsSNPs also called missense 

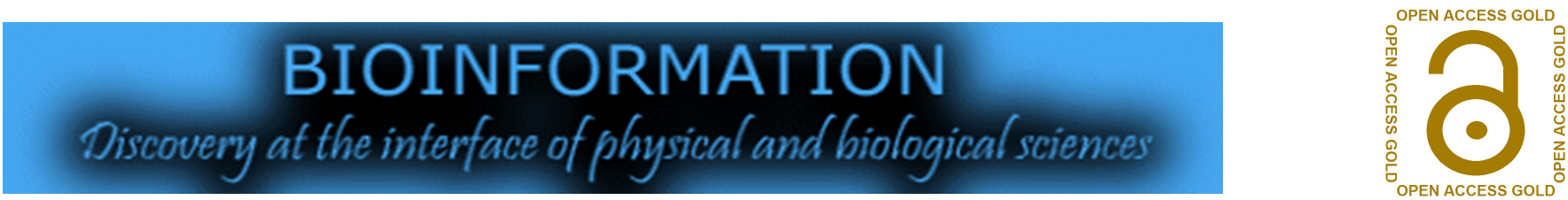

variants, alter amino acid residue in protein sequence and thus change the function of protein through altering protein activity, solubility and protein structure. Nonsense SNPs introduce premature termination in the protein sequence. SNPs have been emerged as the genetic markers for diseases and there are many SNPs markers available in the public databases. With recent advances in high-throughput sequencing technology, many new SNPs have been mapped to human LSP1genes. However, not all SNPs are functionally important. Despite extensive studies of LSP1 proteins in human and effect of their polymorphism in diseases, no attempts was made to comprehensively and systematically analyze to establish the functional consequences of SNPs of LSP1 gene. The aim of this study is to identify the high confident pathogenic SNPs of LSP1 gene and determine their functional consequences using computational methods.

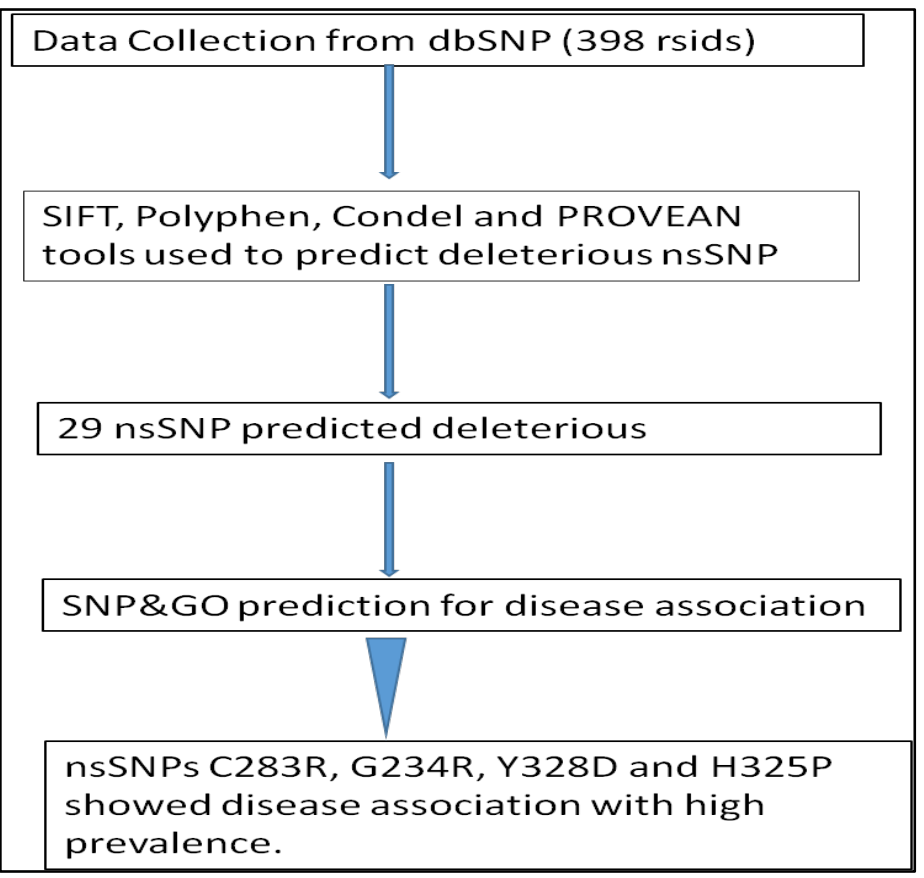

Figure 1: Flow chart depicting overall work methodology adopted in this study.

\section{Materials and Methods \\ SNPs dataset}

The SNPs of the LSP1 (Lymphocyte-specific protein 1) protein were retrieved from the dbSNP database [12]. I used "LSP1" as our search term and filter SNPs. Furthermore, I mapped these SNPs on the genomic coordinate of "NM_002339.2" transcript expresses
LSP1 protein (P33241) for computation analysis of the effect of missense variant. The protein sequences of genes, LSP1 (P33241) was retrieved from the UniProt database [18]. I employed various computational approaches to identify the pathogenic SNPs and their effect on structural and functional consequences of LSP1 (Figure 1)

\section{Tools used for the prediction of SNPs effects}

Predicting deleterious and damaging nsSNPs

SIFT: The algorithm predicted that the tolerant and intolerant coding base substitution based upon properties of amino acids and homology of sequence [13]. The tool considered that vital positions in the protein sequence have been conserved throughout evolution and therefore substitutions at conserved alignment position is expected to be less tolerated and affect protein function than those at diverse positions. I used SIFT version 2.0 [19], which predicted the amino acid substitution score from zero to one. SIFT predicted substituted amino acid as damaging at default threshold score $<0.05$, while score $\geq 0.05$ is predicted as tolerated.

\section{PROVEAN:}

The online tool uses an alignment-based scoring method for predicting the functional consequences of single and multiple amino acid substitutions, and in-frame deletions and insertions [14]. The tool has a default threshold score, i.e. -2.5, below which a protein variant is predicted as deleterious, and above that threshold, a protein variant is neutral.

\section{Condel (CONsensus DELeteriousness):}

This tool evaluates the probability of missense single nucleotide variants (SNVs) deleterious. it computes a weighted average of the scores of SIFT, PolyPhen2, Mutation Assessor and FatHMM [15].

\section{PolyPhen-2:}

This tool is predicting the structural and functional consequences of a particular amino acid substitution in human protein [16]. Prediction of PolyPhen-2 server [20] is based on a number of features including information of structural and sequence comparison. The PolyPhen-2 score varies between 0.0 (benign) to 10.0 (damaging). The PolyPhen-2 prediction output categorizes the SNPs into three basic categories, benign (score $<0.2$ ), possibly damaging, (score between 0.2 and0.96), or probably damaging (score $>0.96$ ).

\section{Predicting disease associated nsSNPs \\ SNPs \& GO:}

A web server predicting whether an amino acid substitution is associated to a disease or not [17]. It is a SVM (Support Vector 


\section{BIOINFORMATION \\ Discovery at the interface of physical and biological science.}

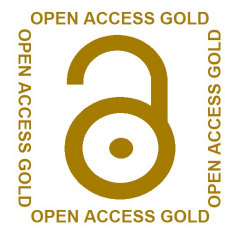

Machine) based tool which takes features of protein sequence, evolutionary information, and functional annotation according to Gene Ontology terms. Isoform 1 of Swiss-Prot Code of LSP1 (P33241) was used and provided the list of amino acid mutations. The results predicted the probability for the polymorphisms of helicase whether being disease- associated or not by three methods: (a) SNPs \& GO, (b) PhD-SNP, and (c) PANTHER. Probability score $>0.5$ is predicted as disease associated variation.

\section{Results and Discussion:}

398rsIDof nsSNPs mapped in human LSP1 gene was downloaded from dbSNP database of NCBI(Table 3), after filtering variation class SNV and function class missense, there were 9590 SNPs mapped to intron, while 457SNPs mapped to 5'UTR, 134SNPs mapped to 3'UTR and 10815 mapped to total SNPs of different variation class (Figure 2). Some rsIDs are associated with multiple SNPs and therefore fall in different classes.

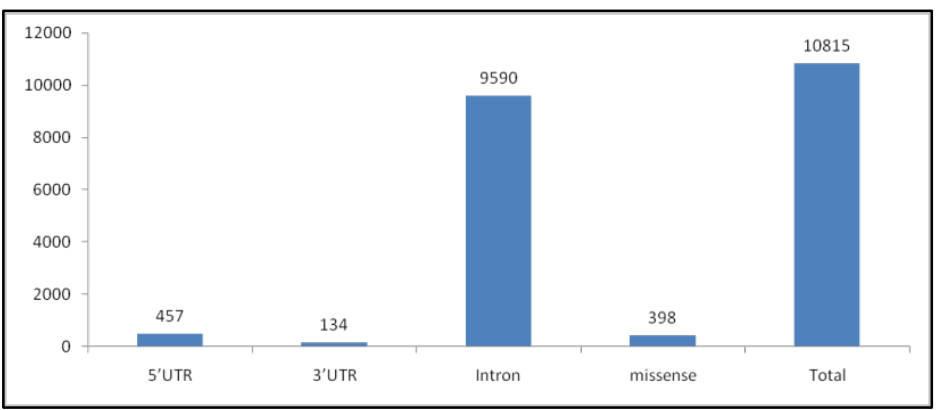

Figure 2: Number of SNPs in different function class of LSP1 gene of human from dbSNP database

\section{Predicting deleterious and damaging nsSNPs}

In order to predict the damaging or deleterious nsSNPs multiple consensus tools were employed. Initially, online tool VEP was used [21]. VEP advantages include: it uses latest human genome assembly GRCh38.p10, and can predict thousands of SNPs from multiple tools including SIFT, Condel, and PolyPhen-2, at a time. 398 nsSNP accession numbers were uploaded to VEP tool and the prediction results were taken for further analysis.

300 missense SNPs was mapped to NM_002339.2 on default scores of consensus tools based on sequence and structure homology methods: (a) SIFT (score <0.5) and (b) PROVEAN (score <-2.5) and Condel (score $>0.522$ ). In order to get a very high confident nsSNPs impacting structure and function of LSP1, I considered high stringent scores across different consensus tools. At parameters of SIFT (score $=0$ ), Polyphen (score >0.96) and Condel (score >0.9), I got 40 nsSNPs (Table 1). These 40nsSNPs were further analyzed by
PROVEAN, which gave 29 nsSNP at default cutoff at -2.5 score fall in the predicted category of deleterious and have damaging effect on protein structure and function (Table 1).

Table 1: List of 40 deleterious missense SNPs on the LSP1 gene identified using prediction tools such as SIFT (score $=0$ ), Condel (score $>0.9$ ), Polyphen (score $>0.96$ ) and PROVEAN (score $=-2.5$ ).

\begin{tabular}{|c|c|c|c|c|c|}
\hline SNP ids & $\begin{array}{l}\text { AA } \\
\text { Change }\end{array}$ & SIFT (score) & Polyphen (score) & Condel (score) & PROVEAN \\
\hline rs757274538 & E74Q & deleterious $(0)$ & probably_damaging(0.924) & deleterious $(0.818)$ & Neutral \\
\hline rs1427708683 & D78N & deleterious $(0)$ & probably_damaging $(0.932)$ & deleterious $(0.823)$ & Deleterious \\
\hline rs371381465 & E79K & deleterious $(0)$ & probably_damaging $(0.934)$ & deleterious $(0.825)$ & Neutral \\
\hline $\mathrm{rs} 371381465$ & E79Q & deleterious $(0)$ & probably_damaging $(0.946)$ & deleterious $(0.835)$ & Neutral \\
\hline rs767014224 & S177N & deleterious $(0)$ & probably_damaging $(0.961)$ & deleterious $(0.849)$ & Neutral \\
\hline rs148262402 & D200Y & deleterious $(0)$ & probably_damaging $(0.963)$ & deleterious $(0.850)$ & Deleterious \\
\hline rs764746759 & R207P & deleterious $(0)$ & probably_damaging $(0.963)$ & deleterious $(0.850)$ & Deleterious \\
\hline rs1347663065 & S212R & deleterious $(0)$ & probably_damaging $(0.963)$ & deleterious $(0.850)$ & Deleterious \\
\hline rs1172211080 & S214R & deleterious $(0)$ & probably_damaging $(0.972)$ & deleterious $(0.859)$ & Deleterious \\
\hline rs1225441968 & $\mathrm{Q} 219 \mathrm{H}$ & deleterious $(0)$ & probably_damaging $(0.973)$ & deleterious $(0.859)$ & Neutral \\
\hline rs1321265627 & L222S & deleterious $(0)$ & probably_damaging $(0.977)$ & deleterious $(0.863)$ & Neutral \\
\hline rs1223328434 & P223R & deleterious $(0)$ & probably_damaging $(0.977)$ & deleterious $(0.863)$ & Deleterious \\
\hline rs1482882164 & S225F & deleterious $(0)$ & probably_damaging $(0.977)$ & deleterious $(0.863)$ & Deleterious \\
\hline rs 375066461 & $\mathrm{I} 227 \mathrm{~V}$ & deleterious $(0)$ & probably_damaging $(0.98)$ & deleterious $(0.869)$ & Neutral \\
\hline rs746869893 & I227T & deleterious $(0)$ & probably_damaging $(0.984)$ & deleterious $(0.875)$ & Deleterious \\
\hline rs769418125 & E232G & deleterious $(0)$ & probably_damaging $(0.985)$ & deleterious $(0.877)$ & Deleterious \\
\hline rs1163688948 & Q233K & deleterious $(0)$ & probably_damaging $(0.987)$ & deleterious $(0.881)$ & Deleterious \\
\hline rs1366846876 & $\mathrm{Q} 233 \mathrm{R}$ & deleterious $(0)$ & probably_damaging( 0.99$)$ & deleterious $(0.886)$ & Deleterious \\
\hline rs748573553 & T235I & deleterious $(0)$ & probably_damaging $(0.99)$ & deleterious $(0.886)$ & Deleterious \\
\hline rs775207068 & T235P & deleterious $(0)$ & probably_damaging $(0.99)$ & deleterious $(0.886)$ & Deleterious \\
\hline $\mathrm{rs} 375475958$ & E239K & deleterious $(0)$ & probably_damaging $(0.991)$ & deleterious $(0.889)$ & Deleterious \\
\hline rs767390484 & R249S & deleterious $(0)$ & probably_damaging $(0.992)$ & deleterious $(0.892)$ & Deleterious \\
\hline rs1392782919 & T263N & deleterious $(0)$ & probably_damaging $(0.994)$ & deleterious $(0.897)$ & Deleterious \\
\hline rs771463495 & T269R & deleterious $(0)$ & probably_damaging $(0.995)$ & deleterious $(0.902)$ & Deleterious \\
\hline rs1263005551 & S276Y & deleterious $(0)$ & probably_damaging $(0.995)$ & deleterious $(0.902)$ & Deleterious \\
\hline rs1263005551 & S276C & deleterious $(0)$ & probably_damaging $(0.996)$ & deleterious $(0.906)$ & Deleterious \\
\hline rs760554324 & C283R & deleterious $(0)$ & probably_damaging $(0.996)$ & deleterious $(0.906)$ & Deleterious \\
\hline rs1327088229 & L296H & deleterious $(0)$ & probably_damaging $(0.996)$ & deleterious $(0.906)$ & Deleterious \\
\hline rs757906951 & W297S & deleterious $(0)$ & probably_damaging $(0.997)$ & deleterious $(0.911)$ & Deleterious \\
\hline rs767954738 & E298K & deleterious $(0)$ & probably_damaging $(0.997)$ & deleterious $(0.911)$ & Neutral \\
\hline rs1203026216 & G301R & deleterious $(0)$ & probably_damaging $(0.998)$ & deleterious $(0.919)$ & Deleterious \\
\hline rs556754848 & G315R & deleterious $(0)$ & probably_damaging $(0.998)$ & deleterious $(0.919)$ & Deleterious \\
\hline rs1345247398 & $\mathrm{K} 316 \mathrm{Q}$ & deleterious $(0)$ & probably_damaging $(0.998)$ & deleterious $(0.919)$ & Neutral \\
\hline rs974685665 & Y318C & deleterious $(0)$ & probably_damaging $(0.998)$ & deleterious $(0.919)$ & Deleterious \\
\hline rs758730712 & K319T & deleterious $(0)$ & probably_damaging $(0.998)$ & deleterious $(0.919)$ & Deleterious \\
\hline rs5578141909 & V321L & deleterious $(0)$ & probably_damaging $(0.998)$ & deleterious $(0.919)$ & Neutral \\
\hline $\mathrm{rs} 1490256278$ & V321A & deleterious $(0)$ & probably_damaging $(0.999)$ & deleterious $(0.935)$ & Neutral \\
\hline rs745616898 & G324R & deleterious $(0)$ & probably damaging $(0.999)$ & deleterious $(0.935)$ & Deleterious \\
\hline rs1468912408 & H325P & deleterious $(0)$ & probably damaging $(0.999)$ & deleterious $(0.935)$ & Deleterious \\
\hline rs1409361986 & Y328D & deleterious $(0)$ & probably_damaging(0.999) & deleterious $(0.935)$ & Deleterious \\
\hline
\end{tabular}

\section{Identifying disease associated nsSNPs}

Furthermore, 29 selected amino acid substitutions in LSP1 protein were used to analyze for disease association. LSP1 Protein ID "P33241" isoform-1and its amino acid mutations were submitted to "SNPs \& GO" tool [22] and the predicted disease association from three different tools were analyzed. The output of (a) SNPs \& GO predicted 4SNPsC283R, G324R, Y328D and H325P are associated with disease and (b) PhD-SNP predicted 14 SNPsR207P, I227T, Q233R, Q233K, T235I, T235P, E239K, C283R, W297S, Y328D, Y318C, K319T, G324R,H325P are associated with diseases, while (c) PANTHER predicted 4 SNPs C283R, L296H, S276C and G301R as disease associated (Table 2). 


\section{BIOINFORMATION \\ Discovery at the interface of physical and biological science.}

Table 2: Prediction of disease associated amino acid substitution using SNPs \& GO, PhD-SNP and PNTHER on29 deleterious or damaging missense SNP using tools such as SIFT, Condel, Polyphen and PROVEAN

\begin{tabular}{|c|l|l|l|c|}
\hline SNP ids & AA Change & PhD-SNP & PANTHER & SNPs\&GO \\
\hline rs1427708683 & D78N & Neutral & Unclassified & Neutral \\
\hline rs148262402 & D200Y & Neutral & Unclassified & Neutral \\
\hline rs764746759 & R207P & Disease & Unclassified & Neutral \\
\hline rs1347663065 & S212R & Neutral & Unclassified & Neutral \\
\hline rs1172211080 & S214R & Neutral & Unclassified & Neutral \\
\hline rs1223328434 & P223R & Neutral & Unclassified & Neutral \\
\hline rs1482882164 & S225F & Neutral & Unclassified & Neutral \\
\hline rs746869893 & I227T & Disease & Unclassified & Neutral \\
\hline rs769418125 & E232G & Neutral & Unclassified & Neutral \\
\hline rs1163688948 & Q233K & Disease & Unclassified & Neutral \\
\hline rs1366846876 & Q233R & Disease & Unclassified & Neutral \\
\hline rs748573553 & T235I & Disease & Unclassified & Neutral \\
\hline rs775207068 & T235P & Disease & Unclassified & Neutral \\
\hline rs375475958 & E239K & Disease & Unclassified & Neutral \\
\hline rs767390484 & R249S & Neutral & Neutral & Neutral \\
\hline rs1392782919 & T263N & Neutral & Neutral & Neutral \\
\hline rs771463495 & T269R & Neutral & Neutral & Neutral \\
\hline rs1263005551 & S276Y & Neutral & Neutral & Neutral \\
\hline rs1263005551 & S276C & Neutral & Disease & Neutral \\
\hline rs760554324 & C283R & Disease & Disease & Disease \\
\hline rs1327088229 & L296H & Neutral & Disease & Neutral \\
\hline rs757906951 & W297S & Disease & Neutral & Neutral \\
\hline rs1203026216 & G301R & Neutral & Disease & Neutral \\
\hline rs556754848 & G315R & Neutral & Unclassified & Neutral \\
\hline rs974685665 & Y318C & Disease & Unclassified & Neutral \\
\hline rs758730712 & K319T & Disease & Unclassified & Neutral \\
\hline rs745616898 & G324R & Disease & Unclassified & Disease \\
\hline rs1468912408 & H325P & Disease & Unclassified & Disease \\
\hline rs1409361986 & Y328D & Disease & Unclassified & Disease \\
\hline
\end{tabular}

\section{Conclusion}

A comprehensive analysis of SNPs of the human LSP1protein with known disease-associated mutations is reported for the first time. The study identified 29 nsSNPs as highly damaging nsSNPs of the human LSP1protein. These high confident damaging nsSNPs were further analyzed for disease association by manual data mapping. Prediction analysis shows that SNPs C283R, G324Rand H325P and Y328D have high prevalence for disease association. Data implies that the reported nsSNPs could potentially alter structure and hence the function of LSP1 protein resulting in pathogenicity with abnormal symptoms describing the disease states. These nsSNPs were associated with significant pathogenicity pending experiment verification to link disease prevalence.

\section{Acknowledgements:}

This project was not supported by any grants agency. I acknowledge with thanks Deanship of Scientific Research (DSR), at King Abdulaziz University, Jeddah for providing their support. I am also thankful to Dr. Ahmad Firoz, Assistant Professor, Department of Biological Science, King Abdulaziz University, Jeddah, KSA, for his critical reviewing of the manuscript.

\begin{tabular}{|c|c|c|c|c|}
\hline rs621679 & rs565801400 & rs772183681 & rs1202288341 & rs1366951082 \\
\hline $\begin{array}{l}\text { rs1140212 } \\
\end{array}$ & rs567011070 & rs773812500 & rs1203026216 & rs1367148625 \\
\hline $\begin{array}{ll}r s 1803928 \\
\end{array}$ & rs569184113 & rs774174728 & rs1206197758 & rs1371307311 \\
\hline $\begin{array}{l}r 57929248 \\
\end{array}$ & rs570838125 & rs774187451 & rs1206383331 & rs1373484177 \\
\hline $\begin{array}{l}\text { rs7938342 } \\
\end{array}$ & rs573166009 & rs774759615 & rs1208571311 & rs1377557151 \\
\hline rs11545725 & rs574262123 & rs775207068 & rs1209026745 & rs1381324548 \\
\hline rs57352451 & rs574587041 & rs775690374 & rs1211172432 & $\mathrm{rs} 1381440832$ \\
\hline rs138247091 & rs575334014 & rs775783036 & rs1213020747 & rs1385778938 \\
\hline rs138303369 & rs576282068 & rs775796745 & rs1214643505 & $\mathrm{rs} 1390296970$ \\
\hline rs138504655 & rs577178834 & rs777162986 & rs1218116157 & rs1390700870 \\
\hline rs140673005 & rs578141909 & rs777226710 & rs1222043175 & rs1391914838 \\
\hline rs141664313 & rs745616898 & rs777617464 & rs1223328434 & $\mathrm{rs} 1392782919$ \\
\hline rs141902712 & rs746345460 & rs778193946 & rs1224210148 & rs1394437978 \\
\hline rs142354742 & rs746869893 & rs778252754 & rs1225441968 & rs1396783838 \\
\hline rs144778074 & rs747106345 & rs779033742 & rs1226157177 & rs1399794061 \\
\hline rs144840874 & rs747369818 & rs779711392 & rs1227502672 & rs1407439097 \\
\hline rs145216198 & rs747468057 & rs779796182 & rs1232033724 & rs1409361986 \\
\hline rs146468121 & rs747544389 & rs779888159 & rs1233355677 & rs1410605938 \\
\hline $\begin{array}{l}\text { rs147310705 } \\
\end{array}$ & rs747621569 & rs780821356 & rs1234696950 & rs1412542490 \\
\hline rs147890004 & rs747742064 & rs781120168 & rs1240434942 & rs1413977301 \\
\hline rs147990493 & rs748208610 & rs781492964 & rs1241527965 & rs1414831389 \\
\hline rs148042410 & rs748401091 & rs866361186 & rs1242184369 & rs1416863114 \\
\hline rs148262402 & rs748573553 & rs866872817 & rs1243095899 & rs1417172855 \\
\hline rs148966414 & rs749677355 & rs866926158 & rs1243463270 & rs1420092778 \\
\hline rs149086047 & rs750149067 & rs867314806 & & rs1422008007 \\
\hline rs149491406 & rs750915233 & rs868173065 & rs1245841526 & rs1422217064 \\
\hline rs150432651 & rs750992011 & $\mathrm{rs} 868500426$ & rs1247334626 & rs1423047689 \\
\hline rs150456040 & rs751107694 & rs878889192 & & $\mathrm{rs} 1423202063$ \\
\hline rs150542237 & rs751527292 & rs879106981 & rs1249156883 & rs1427708683 \\
\hline rs181774507 & rs752408075 & rs887699875 & rs1250098025 & rs1430559453 \\
\hline rs182693925 & rs753356088 & rs888898118 & rs1250264665 & $\mathrm{rs} 1430649392$ \\
\hline rs184276196 & rs753582906 & rs891974211 & rs1250725212 & rs1434072090 \\
\hline rs189506078 & rs754249948 & rs892720144 & rs1251749609 & rs1435814360 \\
\hline rs199756727 & rs754745738 & rs895629191 & rs1254008276 & rs1437946454 \\
\hline rs199783035 & rs755253787 & & & \\
\hline rs200019612 & rs755491188 & rs910560883 & rs1263005551 & rs1445305286 \\
\hline rs200067113 & rs755782795 & rs918757420 & rs1265743121 & rs1446623366 \\
\hline rs200522804 & rs756566635 & $\mathrm{rs} 923411713$ & rs1267291484 & rs1446638347 \\
\hline rs200748215 & rs757274538 & & & rs1448500435 \\
\hline rs201040841 & $\begin{array}{rr}r 5757527171 \\
\end{array}$ & rs945925029 & rs1270942861 & rs1448961397 \\
\hline rs201670929 & rs757725608 & rs 945952465 & rs1274339078 & rs1449103483 \\
\hline rs202240419 & & & & rs1450439364 \\
\hline rs267602812 & rs758125057 & rs952815816 & rs1282961531 & rs1452607509 \\
\hline rs368052660 & rs758730712 & rs952911063 & rs1285324855 & rs1452916657 \\
\hline rs368065769 & rs759191270 & rs959933777 & rs1291913683 & rs 1453014034 \\
\hline rs368886999 & & & rs1293971 & \\
\hline rs369531651 & rs760554324 & rs974685665 & rs1294666770 & rs1457081847 \\
\hline rs369993990 & rs760660868 & rs986034440 & rs1295737723 & rs1460940380 \\
\hline rs370562045 & & $\begin{array}{ll}\mathrm{rs} 998 \\
\end{array}$ & $\begin{array}{ll}\mathrm{rs} 1298 \\
\end{array}$ & rs1462548468 \\
\hline rs370626038 & & rs1000519442 & rs129 & rs1464317130 \\
\hline rs371381465 & 76917 & 838 & rs1299 & rs1465757982 \\
\hline rs371615334 & rs762959419 & 657306 & rs1303610213 & rs1466007447 \\
\hline rs37203091 & & & & 909235 \\
\hline rs372146610 & $\begin{array}{l}\text { rs763948767 } \\
\end{array}$ & 94 & 503 & rs1468535644 \\
\hline rs 3724500 & & & & rs1468912408 \\
\hline rs373309025 & $\frac{1507}{\text { rs764 }}$ & & & $\mathrm{rs} 1471227409$ \\
\hline rs373401268 & 46759 & & 1321122730 & rs1473317251 \\
\hline rs373858328 & rs764 & 1044635385 & 265627 & rs1474105982 \\
\hline rs 3744066 & rs764 & & rs1323 & rs1475328014 \\
\hline rs 375 & & & rs1327088229 & rs1475667216 \\
\hline rs375326868 & rs765491711 & 576776 & rs1332594197 & rs1479358037 \\
\hline rs375475958 & rs766 & (9958990 & rs1333563092 & rs1482882164 \\
\hline rs376029050 & rs766651093 & rs1163688948 & rs1334846403 & rs 1484168260 \\
\hline rs 376 & & & & \\
\hline & & & & rs1486073931 \\
\hline rs527743009 & rs767014224 & $\begin{array}{lr}\text { rs1170512001 } \\
\end{array}$ & rs1337630668 & rs1490256278 \\
\hline rs530862911 & rs767061907 & $\mathrm{rs} 1172211080$ & rs1339506361 & rs1490261047 \\
\hline rs534563533 & rs767390484 & & rs1340203839 & rs1565074079 \\
\hline & & & & \\
\hline rs538542793 & rs768294917 & 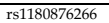 & rs1345247398 & rs1565085055 \\
\hline rs539714151 & & & rs1347018258 & rs1565085108 \\
\hline & & & rs1347663065 & rs1565086802 \\
\hline rs553028792 & rs769962820 & $\begin{array}{ll}\text { rs1187059148 } \\
\end{array}$ & rs1349693890 & rs57739592 \\
\hline rs556754848 & rs770047466 & rs1189732756 & rs1349980392 & rs17855362 \\
\hline rs557026040 & & rs1192423892 & & \\
\hline rs558867326 & & & rs1358207243 & rs3188464 \\
\hline rs561026287 & rs770351321 & rs1194011645 & rs1358988213 & rs186236551 \\
\hline rs563563171 & rs771463495 & rs1194094051 & rs1361728086 & rs57757026 \\
\hline rs564056573 & rs771507322 & & rs1366398226 & \\
\hline rs564198572 & rs772024277 & rs1201337942 & rs1366846876 & \\
\hline
\end{tabular}




\section{BIOINFORMATION \\ Discovery at the interface of physical and biological sciences}

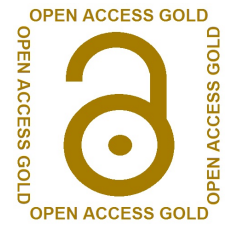

\section{References:}

[1] https://www.ncbi.nlm.nih.gov/gene/4046

[2] Jongstra-Bilen J et al. Blood 2000 96:1827 [PMID 10961883].

[3] Palker TJ et al. Hybridoma 1998 17:497 [PMID 9890705]

[4] Pulford K et al. Immunology 1999 96:262 [PMID 10233704]

[5] Howard TH et al. Blood 1998 91:4786 [PMID 9616178]

[6] Tang Z et al. Nucleic Acids Res. 2017 45:W98 [28407145]

[7] Bhattacharya A et al. Nucleic Acids Res. 2014 42:D86 [PMID 24163105].

[8] Wang Z \& Moult J. Hum Mutat. 2001 17:263 [PMID11295823].

[9] Bromberg Y \& Rost B. BMC Bioinformatics 2009 10:S8 [PMID 19758472].

[10] Calabrese R et al. Hum Mutat 2009 30:1237 [PMID 19514061].

[11] Bao Let al. Nucleic Acids Res 2005 33:W480 [PMID 15980516].

[12] Sherry ST et al. Nucleic Acids Res 2001 29:308 [PMID 11125122].
[13] Sim NL et al. Nucleic Acids Res 201240 W452 [PMID 22689647].

[14] Choi Y \& Chan AP. Bioinformatics 2015 31(16) 2745 [PMID 25851949].

[15] Adzhubei IA et al. Nat Methods 2010 7:248 [PMID 20354512].

[16] Hecht M et al. BMC Genomics 2015 16: S1 [PMID 26110438 ].

[17] Calabrese R et al. 2009 Human Mutation 30:1237 [PMID 19514061].

[18] https://www.uniprot.org

[19] http://sift.jcvi.org/

[20] http://genetics.bwh.harvard.edu/pph2/

[21] http://www.ensembl.org/Tools/VEP

[22] http://snps.biofold.org/snps-and-go/snps-and-go.html

Edited by P Kangueane

Citation: Ali, Bioinformation 15(9): 621-626 (2019)

License statement: This is an Open Access article which permits unrestricted use, distribution, and reproduction in any medium, provided the original work is properly credited. This is distributed under the terms of the Creative Commons Attribution License 


\section{BIOINFORMATION}

Discovery at the interface of physical and biological sciences
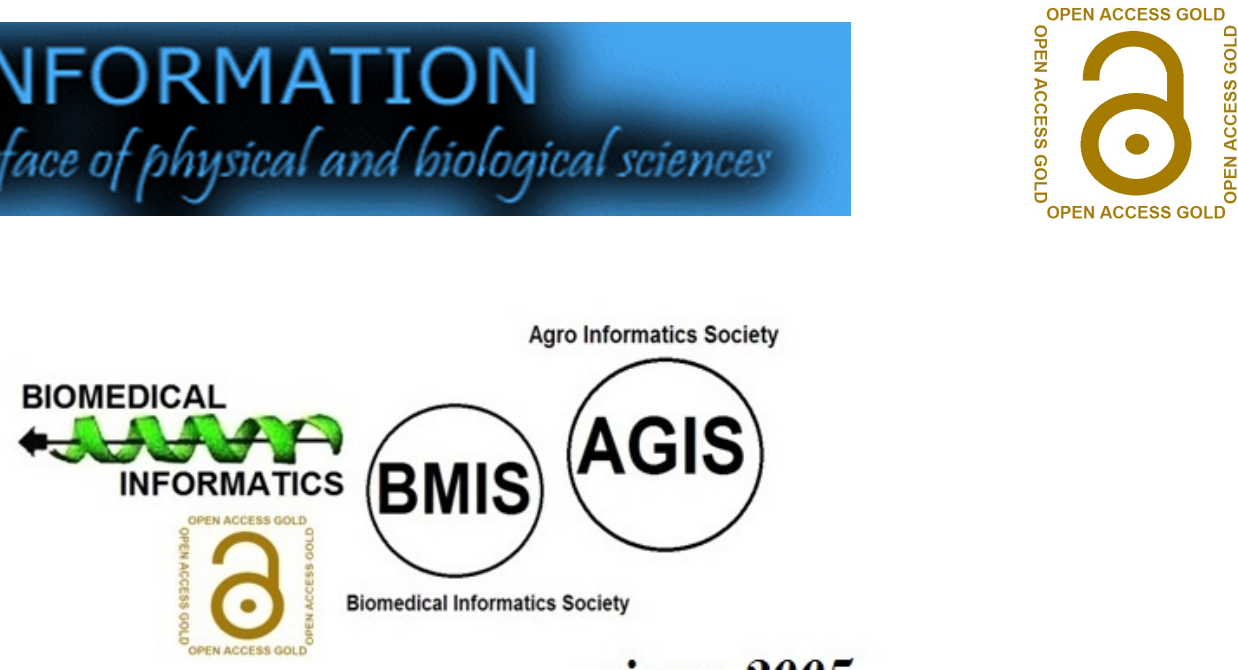

since 2005

\section{BIOINFORMATION}

Discovery at the interface of physical and biological sciences

\section{indexed in}

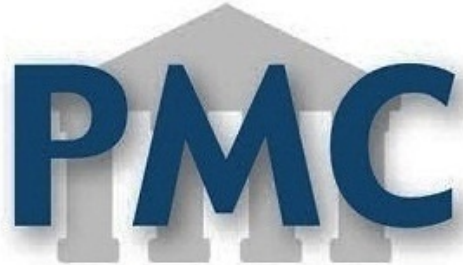

PublMed

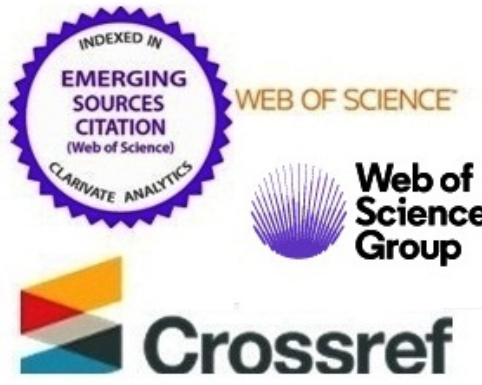

EBSCO

Web of

Science

roup

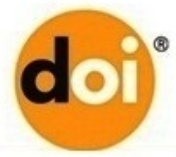

ResearchGate
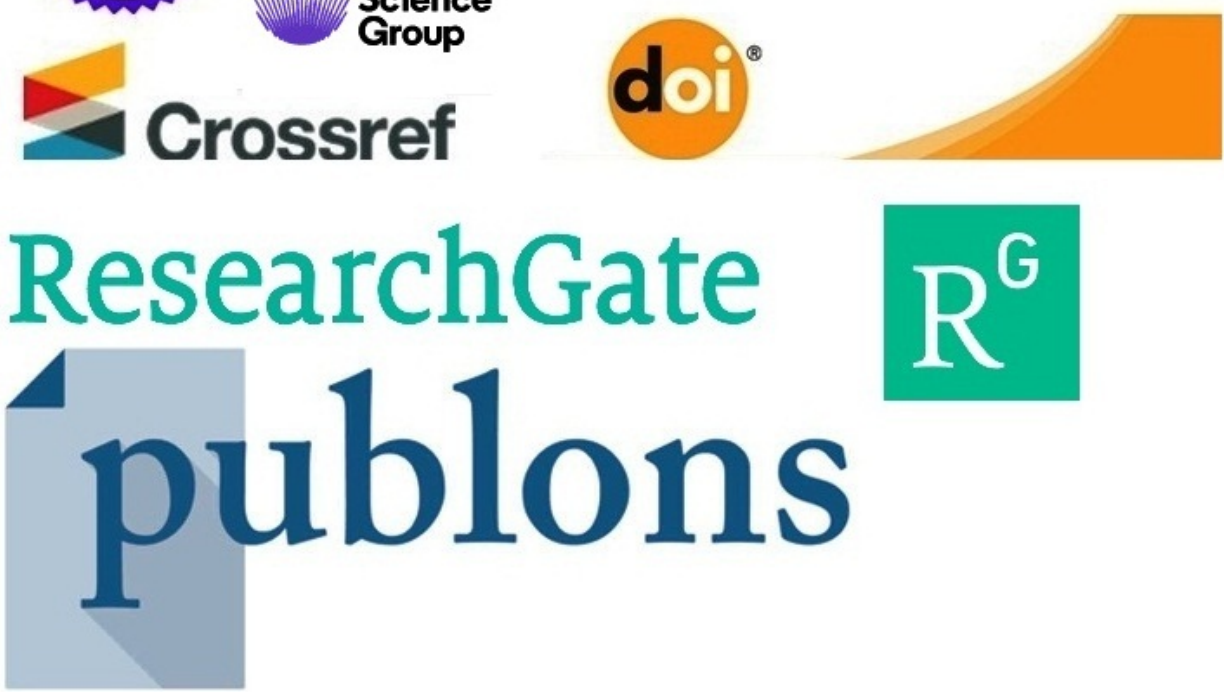

ISSN 0973-2063 (online) 0973-8894 (print) 